\title{
Atomistic insight into the adsorption site selectivity of stepped Au(111) surfaces
}

\author{
Roberto Gaspari, Carlo A. Pignedoli, Roman Fasel, Matthias Treier, and Daniele Passerone* \\ Empa, Swiss Federal Laboratories for Materials Science and Technology, nanotech@surfaces laboratory, Ueberlandstrasse 129, \\ CH-8600 Dübendorf, Switzerland
}

(Received 10 June 2010; published 21 July 2010)

\begin{abstract}
Using classical and ab initio simulations, we study the interplay between the $\mathrm{Au}(111)$ surface reconstruction and monoatomic steps on a vicinal face. The experimentally observed discommensuration line patterns on a specific vicinal are reproduced and explained, and a complete description of the structure is given. An unusual atomic arrangement is shown to be responsible for the lower reactivity of hep segments of step edges compared to the one of fcc segments. Our results provide an unprecedented understanding of the electronic and geometric properties of the complex $\mathrm{Au}(111)$ surface.
\end{abstract}

DOI: 10.1103/PhysRevB.82.041408

PACS number(s): 68.35.B-, 31.15.A-, 68.37.Ef, 68.47.De

Gold provides outstanding examples of surface reconstruction on all its high symmetry faces. Among them, $\mathrm{Au}(111)$ has lately gained renewed interest due to the role as template played by its herringbone reconstruction in the field of molecular nanoscience. ${ }^{1}$ On $\mathrm{Au}(111)$ the increase in the surface atomic density along one close-packed direction leads to a pattern of alternating bulklike fcc and faulted hcp stacking at the surface, the so-called $22 \times \sqrt{3}$ reconstruction. ${ }^{2,3}$ Surprisingly, the subtle difference in atomic stacking between hcp and fcc regions induces a clear selectivity with respect to adsorption. ${ }^{4}$ Molecules generally prefer fcc regions but there is an even "better" place for them to adsorb on, namely, at a surface step, which has a lower work function, exposes available electronic density, and is willing to coordinate incoming species. In this Rapid Communication we fill the lack of understanding about the interplay between steps and reconstruction by means of large scale $a b$ initio simulations.

Steps are always present in low concentration at real nominally flat surfaces but they can also appear as an array regularly spaced by flat terraces in an atomically miscut $\mathrm{Au}(111)$, called vicinal surface. Thus, with the rise of surface nanoscience, stepped vicinal surfaces have become one of the templates of choice for the production of regularly arranged molecular structures. In the case of $\mathrm{Au}(111)$ vicinals, molecules not only adsorb at steps but they decorate them in a precise order, dependent on the local stacking (fcc or hcp). At a vicinal surface, as in the case of $\mathrm{Au}(677)$ or $\mathrm{Au}(1112$ $12)$, the $22 \times \sqrt{3}$ pattern is slightly modified by the step and, along the terraces, hcp- and fcc-stacking regions form $\mathrm{V}$-shaped domains separated by ridges standing out of the surface. The (lateral) atomic structure of the surface far away from step edges can be accurately studied by scanning tunneling microscopy (STM) (Refs. 3, 5, and 6) but at proximity of steps, electron tunneling from the lower terrace and the near step cannot be trivially decoupled. Therefore much of the structural information on the atomic arrangement at step edges is lost; in particular, the way the reconstructed terrace turns into bulk at the lower step edge (LSE). This has hitherto prevented a detailed understanding of atomic and molecular self-assembly phenomena on vicinal $\mathrm{Au}(111)$ surfaces that have highlighted a striking adsorption site selectivity. ${ }^{7-11}$ For instance, polycyclic aromatic hydrocarbons preferen- tially adsorb on kinks, then on step lines facing fcc regions and finally on those facing hcp ones ${ }^{8,9,12}$ and a similar behavior is observed for $\mathrm{C}_{60} \cdot{ }^{11}$ For the adsorption of 1-nitronaphthalene on $\mathrm{Au}(111)$, a tentative explanation of the step selectivity was proposed by Vladimirova et al. ${ }^{13}$ who performed density-functional-theory (DFT) calculations which assumed that the region beneath the step was not reconstructed. In this Rapid Communication we present instead the results of large scale calculations performed without any assumptions about the step structure: the latter is obtained, using a full model of the vicinal reconstructed surface.

Our results show that an unprecedented atomic rearrangement appears at hcp-stacking areas of the step, where the atoms form a local close-packed plane and the step edge is dramatically smoothed. The structural differences between the hcp and the fcc step regions are assessed through comparison with low-temperature STM data. Electric field calculations along the step edge, as well as adsorption energy calculations for benzene on the fcc and hcp parts of the steps, confirm a one-to-one correspondence between the atomic geometry of the step segments and the observed adsorption site selectivity.

We focus on the interplay between steps and the $22 \times \sqrt{3}$ reconstruction, by considering the $\mathrm{Au}(677)$ surface that experimentally has the shortest terraces among all $\mathrm{Au}(111)$ vicinals displaying reconstruction. Experimentally, the presence of steps leads to a reconstruction periodicity slightly larger than that typical of the $22 \times \sqrt{3}$ pattern, due to an effect of stress relief. 6,14 Here, instead, we consider the $\mathrm{Au}(677)$ surface with an atomic density corresponding to the $22 \times \sqrt{3}$ reconstruction, in analogy to the flat reconstructed $\mathrm{Au}(111)$. We first perform annealing simulations and structural optimizations of a slab with a monoatomic kink superlattice using the Ercolessi-Parrinello-Tosatti (EPT) manybody "glue potential" 15 and the IMD simulation package. ${ }^{16}$ The EPT potential is chosen since it predicts the $22 \times \sqrt{3}$ pattern to be a stable configuration at $0 \mathrm{~K} .{ }^{17}$ Our simulation cell encompasses three periods of the $22 \times \sqrt{3}$ superlattice, three steps, and 18 layers. A kink superlattice comparable to the one experimentally studied by Leroy et al. ${ }^{14}$ is obtained by tilting the unit cell of a kink-free $\mathrm{Au}(677)$ by a suitable angle which corresponds to one kink per unit cell (kink-kink 
distance of $6.3 \mathrm{~nm}$ ). Results show that a unit cell including 1 period of the $22 \times \sqrt{3}$ superlattice, one step and six layers is large enough to accurately describe the surface relaxation. With such unit cell, the best obtained EPT configuration from slab optimizations is used as starting point for full $a b$ initio optimizations using DFT within the pseudopotential approach, employing the mixed Gaussian and plane-wave code $\mathrm{CP} 2 \mathrm{~K} .{ }^{18,19}$ Constant-current STM images were acquired under ultrahigh vacuum conditions at a sample temperature of $5.7 \mathrm{~K}$ using an Omicron low temperature STM. We used a $\mathrm{Au}(11,12,12)$ single crystal (Surface Prep. Laboratory, Netherlands) that was cleaned by standard sputtering-annealing cycles.

In order to propose a correct atomistic model the first task is to understand whether the density increase obtained through reconstruction encompasses the whole terrace up to the LSE. Surface free energies of EPT-optimized Au(111) vicinal models are compared as a function of the different number of reconstructed rows on a terrace; using the cohesive energy of gold as the chemical potential we find that the most stable state is the one where the reconstruction extends up to the step edge, i.e., in the case of $\mathrm{Au}(677), 14$ rows are uniaxially compressed according to the $22 \times \sqrt{3}$ reconstruction; ${ }^{20}$ this behavior is different to what observed, for example, in $\mathrm{Au}(100)$, where some rows close to the step edge are bulk terminated. ${ }^{21}$ The presence of discommensuration lines on $\mathrm{Au}(111)$ surfaces is usually taken as an evidence for the existence of regions with different atomic stackings. In our simulations, we assign to each surface atom a "hcp" or "fcc" label if its lateral distance to a reference atom in the third (hcp) or fourth (fcc) layer is smaller than a threshold $d_{l}=0.56 \AA$, taken as one third of the distance between two neighboring hollow sites. A contour map of the physical corrugation together with the stacking of atoms is shown in Figs. 1(a) and 1(b).

The V-shaped discommensuration line pattern observed experimentally is clearly reproduced. Surface atoms within domains that are widest at the LSE are in fcc stacking positions, whereas domains widest at the upper step edge (USE) contain hcp-stacked atoms. The surface corrugation at the center of the terrace is very similar to that of the flat $\mathrm{Au}(111)$ reconstruction pattern and displays a maximum excursion of $0.14 \AA$ and a height difference of about $0.05 \AA$ between hcp and fcc regions. Atomic rows near the center of the terrace undulate laterally with an amplitude of $0.7 \AA$ and the kink site appears exactly at the center of the fcc domain. All these features perfectly match experimental findings., ${ }^{4,5,14,22} \mathrm{~A}$ closer look reveals that at the LSE the terrace atoms match the bulk structure and the fcc regions are dominant. However, the atomic mismatch between the reconstructed terrace and the second layer [Figs. 2(a) and 2(c), orange (dark gray) and purple (black) lines) makes it impossible to maintain a triangular lateral packing everywhere along the LSE. This drives some atoms of the LSE into a squared lateral stacking with the neighboring bulk atoms as shown in Figs. 2(a) and 2(c). The V-shaped hcp terrace domains stem out right from these defects while all bulk atoms beyond the LSE sit perfectly in bulklike fcc positions. However, the complex atomic arrangement of the LSE prevents the atoms of the defect to occupy hcp sites. Because of simple geometrical

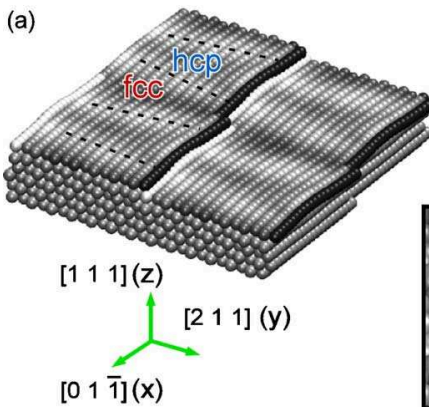

(b)

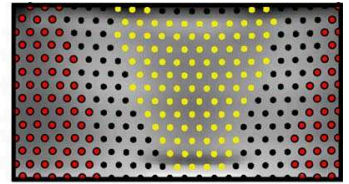

(c)

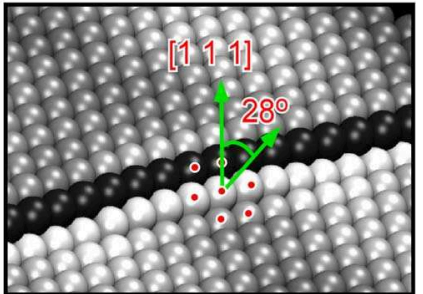

FIG. 1. (Color online) (a) Atomic geometry of the stepped $\mathrm{Au}(677)$ surface including a kink in each unit cell $(2 \times 2 \times 1$ cells are shown) after optimization using DFT. The grayscale reflects the surface corrugation with respect to the average terrace height. $\mathrm{V}$-shaped discommensuration lines separate fcc-from hcp-stacking areas. (b) Top view of the corrugation profile of a single surface unit cell. Largest outward corrugation corresponds to brightest regions. Top layer atom positions are superimposed as dots and colored according to the stacking with respect to the underlying layers: hcp (yellow-bright gray), bridge (black), or fcc (red-dark gray with black border). (c) Close-up view of (a) at the hep part of the step: atoms indicated by red (dark gray) dots are perfectly coplanar, forming a local close-packed facet.

considerations, this would require the LSE atoms to have an excursion in the $y$ direction [Fig. 1(a)] which is hindered by the tendency of LSE rows to straighten out to match the crystallographic bulk structure.

As a general trend, $\mathrm{Au}(111)$ surface atoms relax outwards when they sit in regions of unfavorable stacking. ${ }^{23-25}$ This is also the case of atoms forming the defects at the LSEs that have a very unusual coordination with respect to the underneath layers. On an open terrace, such stacking would lead (within the same level of theory) to an outward relaxation of bare $0.25 \AA$. In the present case, however, the interaction between atoms of the LSE and the USE enhances the outward relaxation of the unfavorably stacked atoms which stand out as much as $1 \AA$ above the underlying terrace level [Fig. 2(c)]. Thus, the large vertical corrugation brings upper and lower edge atoms very close to each other at the hcp regions, driven by yet another mechanism of relaxation, namely, a gain in lateral coordination across the step. After DFT optimization atoms of the upper rim and lower edge of the steps become perfectly planar and close-packed in the hcp region, thereby forming a "local close-packed plane" that is slightly tilted (by $28^{\circ}$ ) with respect to the nominal (111) surface and has the features of a "slide" connecting upper and lower terraces [Fig. 1(c)]. We note that strongly anisotropic geometries have been already reported for gold nanoclusters and the reason for these arrangements has been attributed to relativistic effects ${ }^{26-28}$ inducing significant $s$ - $d$ hybridization and strong directionality effects in bonding.

At the fcc part of the steps, atoms of the USE lean upon fcc atoms of the LSE and the resulting local geometry is therefore very similar to that of a bulk terminated vicinal. The huge structural difference between the hcp and the fcc 


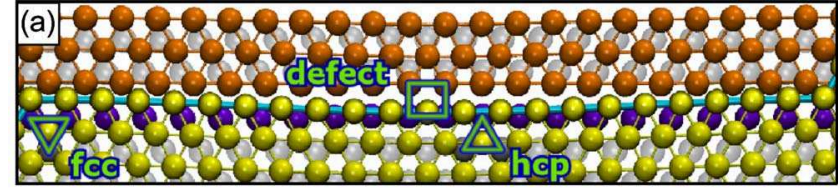

(b)
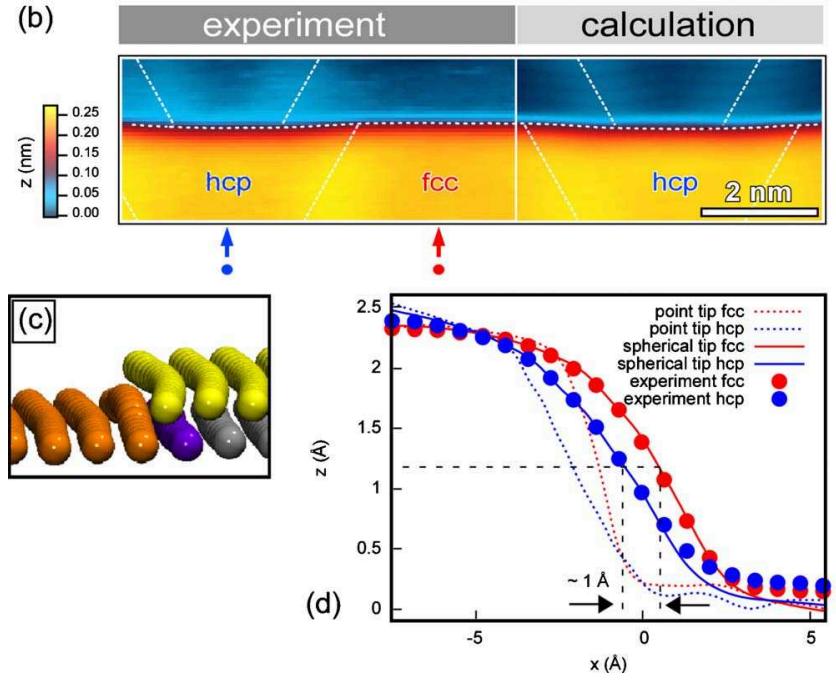

FIG. 2. (Color online) (a) Top view of a part of the DFT optimized $\mathrm{Au}(677)$. Different stacking orders are highlighted. The mismatch between the last LSE (orange-dark gray) line and the first bulk (purple-black) line is evident by the presence of a square arrangement (defect). (b) Closeup of the surface at step edges in experiment $(-50 \mathrm{mV} ; 100 \mathrm{pA})$ and theory. The V-shaped discommensuration lines, together with a large lateral corrugation of the step can be observed. (c) Side view of the same region shown in (a). (d) Profile of the simulated STM image using the Tersoff-Hamann approximation for a point tip and a spherical model tip of radius of $7 \AA$, together with experimental results. Theoretical profiles, obtained with an LDOS integrated up to $-0.1 \mathrm{eV}$, are taken at a charge isovalue of $7 \times 10^{-6} \mathrm{e} / \AA^{3}$.

part of the step is reflected by the integrated local density of states (LDOS) profiles across the two regions. Within the Tersoff-Hamann approximation, LDOS integrated between the Fermi energy and a finite bias can be compared with experimental STM maps of the surface. In our case, the latter have been performed with a very small bias $(-50 \mathrm{meV})$ in order to achieve atomic resolution. Simulated maps are obtained with the lowest possible bias allowed by our calculations $(-0.1 \mathrm{eV})$. In Fig. 2(d) (dashed lines) we report the 7 $\times 10^{-6} \mathrm{e} / \AA^{3}$ isovalue of the Tersoff-Hamann (point tip) across the fcc and the hcp step, showing that the hcp profile is much broader and less steep than the fcc one. This feature is robust on a range of isovalues. By linearly fitting the charge density profile at half height of the step, it is found that the fcc curve has a slope of $45^{\circ}$ with respect to the terrace normal, while the hcp slope amounts to $28^{\circ}$.

The STM profiles across the hcp and the fcc part of the step indeed show features that qualitatively resemble the simulated Tersoff-Hamann (point tip) lines [see Fig. 2(d)]: the slope and the shape of the profiles in the two regions reveal a difference which appears intrinsic to the surface structure.
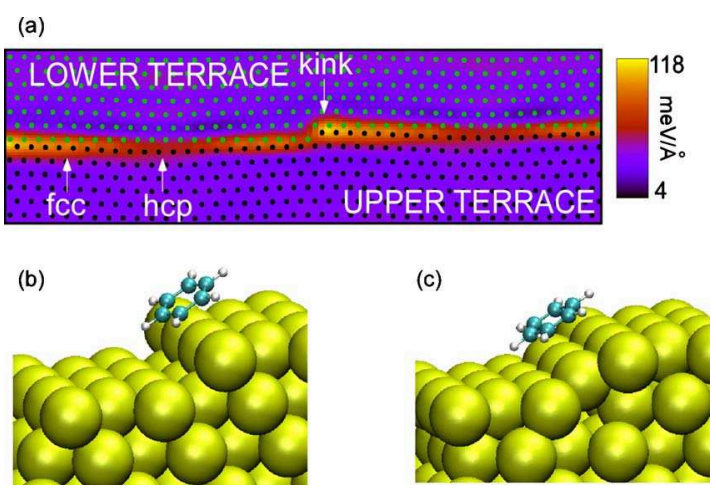

FIG. 3. (Color online) (a) Modulus of the electric field $(|\vec{E}|)$ showing that the largest values are found at the step region. Along the step, the largest values are found at kinks, and the lowest at hcp regions (kink-hcp difference of about $60 \mathrm{meV} / \AA$ ). (b) Adsorption geometry of a benzene molecule on a step with fcc-like structure. (c) The same molecule on a smoothened step in the hcp region.

Nevertheless, the experimental profiles are much broader than the theoretical ones and the difference between hcp and fcc domains much smaller. The slope of the hcp lines is $22^{\circ}$ and that of the fcc lines is about $25^{\circ}$. The reason for this discrepancy must be searched in the finite size of the tip, making the step appear more broadened than in the case of an ideal point probe. In order to quantitatively reproduce the experimental results we used a spherical model tip ${ }^{29}$ and compared our results to the experiments using the sphere radius as a single fitting parameter. For a wide range of reasonable values of the tip radius, we found, as in the experiment, a different shape and slope in the two domains. Using a $7 \AA$ radius sphere we obtain perfect agreement between the simulated and the experimental profiles in the fcc and hcp regions [Fig. 2(d)], which further validates our model.

Finally, it is interesting to note that at half height of the step the experimental lateral excursion of the step is about $1 \AA$ which is larger than that typical of a reconstructed row in the flat $\mathrm{Au}(111)$ surface. This value reflects the reduced coordination of the step atoms and implies a different stacking at the USE, where the hcp regions appear larger than the fcc ones, as also demonstrated by the theoretical and experimental corrugation profile [see Figs. 1(a) and 2(c)].

The dramatic structural differences between fcc and hcp step regions must have a great impact on the selectivity of the corresponding step segments. At hcp regions we do not even have sharp steps anymore but rather a change in the slope of the (111) surface. Therefore, all effects that make steps more reactive than flat regions, such as the Smoluchowski effect and coordination of step atoms, are more loosely expressed in the hcp part of the step than in the fcc one.

We have calculated a quantity that is directly related to the adsorption of polarizable molecules, the modulus of the electric field $|\vec{E}|$ (computed as the gradient of the Hartree plus ionic potential) on an isosurface of the charge density $\rho$. The chosen value of $\rho$ corresponds to a position about $3 \AA$ above the USE, which is a typical adsorption height for molecules on metal surfaces. ${ }^{30,31}$ In a simple picture, where the gradient of the potential is stronger so will be the gain in 
energy of the molecule, that will reorient its induced dipole $\vec{p}=\hat{\alpha} \cdot \vec{E}$ ( $\hat{\alpha}$ being the polarizability) as to lower its internal energy by a quantity (in case of an isotropic $\hat{\alpha}$ ) proportional to $|\vec{E}|^{2}$. As it can be appreciated in Fig. 3 the profile of $|\vec{E}|$ along the terrace shows that kinks are the most reactive parts followed by steps in the fcc regions. Steps in the hcp regions are associated with a lower value of $|\vec{E}|$ and therefore are expected to be less reactive toward polarizable molecules.

As a final example we calculated the adsorption energy difference between a benzene molecule located on the step at a fcc and at a hcp region, using the same DFT scheme with the addition of the van der Waals correction introduced by Grimme. ${ }^{30,32}$ We find that adsorption on the fcc part of the step is more stable by $80 \mathrm{meV}$ than on the "flattened" hcp step [Figs. 3(b) and 3(c)], confirming the prediction of surface selectivity based on the structural properties of the surface.

In summary, we presented an atomistic characterization of $\mathrm{Au}(111)$ vicinals with $\{111\}$-like steps based on DFT simulations and experimental data. Our analysis showed that the
V-shaped pattern of reconstruction observed on vicinals such as the $\mathrm{Au}(677)$ turns into the bulk structure in an abrupt way at the LSEs: the reconstruction is predicted to reach exactly the bottom edge of the terraces, where the interaction between upper and lower edges of the steps at the hcp regions leads to the formation of an unusual structure, i.e., a planar arrangement of atoms that connects the upper and lower terraces of the gold staircase. A calculation of the electric field along the step has shown that such structure is related to a different reactivity toward polarizable molecules of kinks, hcp regions and fcc regions along the steps. Finally, DFT simulations predict the adsorption of benzene to be more stable on the fcc step than on the hcp one by $80 \mathrm{meV}$, confirming the prediction of surface selectivity based on the structural properties of the surface.

We thank Erio Tosatti for illuminating discussions and the Swiss Center for Scientific Computing for computational resources. R.G. thanks the Swiss National Science Foundation for the financial support.

*daniele.passerone@empa.ch

${ }^{1}$ S. Clair, W. Pons, H. Brune, K. Kern, and J. Barth, Angew. Chem., Int. Ed. 44, 7294 (2005).

${ }^{2}$ U. Harten, A. M. Lahee, J. P. Toennies, and C. Wöll, Phys. Rev. Lett. 54, 2619 (1985).

${ }^{3}$ J. Barth, H. Brune, G. Ertl, and R. Behm, Phys. Rev. B 42, 9307 (1990).

${ }^{4}$ L. Bürgi, H. Brune, and K. Kern, Phys. Rev. Lett. 89, 176801 (2002).

${ }^{5}$ Ch. Wöll, S. Chiang, R. J. Wilson, and P. H. Lippel, Phys. Rev. B 39, 7988 (1989).

${ }^{6}$ S. Rousset, V. Repain, G. Baudot, Y. Garreau, and J. Lecoeur, J. Phys.: Condens. Matter 15, S3363 (2003).

${ }^{7}$ M. Böhringer, K. Morgenstern, W.-D. Schneider, M. Wühn, Ch. Wöll, and R. Berndt, Surf. Sci. 444, 199 (2000).

${ }^{8}$ M. Treier and R. Fasel, Chimia 63, 122 (2009).

${ }^{9}$ M. Treier, P. Ruffieux, R. Schillinger, T. Greber, K. Müllen, and R. Fasel, Surf. Sci. 602, L84 (2008).

${ }^{10}$ Y. Makoudi, C. Didiot, F. Palmino, E. Duverger, B. Kierren, D. Malterre, and F. Chérioux, Surf. Sci. 604, 27 (2010).

${ }^{11}$ W. Xiao, P. Ruffieux, K. Aït-Mansour, Gröning, K. Palotas, W. A. Hofer, P. Gröning, and P. Fasel, J. Phys. Chem. B 110, 21394 (2006).

${ }^{12}$ M. Treier, Ph.D. thesis, University of Zurich, 2009.

${ }^{13}$ M. Vladimirova, M. Stengel, A. De Vita, B. A., M. Böhringer, K. Morgenstern, R. Berndt, and W. Schneider, Europhys. Lett. 56, 254 (2001).

${ }^{14}$ F. Leroy, G. Renaud, A. Letoublon, S. Rohart, Y. Girard, V. Repain, S. Rousset, A. Coati, and Y. Garreau, Phys. Rev. B 77, 045430 (2008).

${ }^{15}$ F. Ercolessi, E. Tosatti, and M. Parrinello, Phys. Rev. Lett. 57, 719 (1986).

${ }^{16}$ J. Stadler, R. Mikulla, and H.-R. Trebin, Int. J. Mod. Phys. C 8, 1131 (1997).

${ }^{17}$ F. Ercolessi, A. Bartolini, M. Garofalo, M. Parrinello, and E. Tosatti, Surf. Sci. 189-190, 636 (1987).

${ }^{18}$ J. VandeVondele, M. Krack, F. Mohamed, M. Parrinello, T. Chassaing, and J. Hutter, 2005, http://cp2k.berlios.de/

${ }^{19}$ We use the Perdew-Burke-Ernzerhof exchange-correlation functional using a Goedecker-Teter-Hutter norm-conserving pseudopotential, with a cutoff of $70 \mathrm{Ry}$; the wave function is expanded using Gaussian basis functions of the class DZVP (double zeta valence with polarization). A typical slab used for DFT simulations encompasses six Au layers.

${ }^{20}$ Annealing simulations performed at high temperature, starting from any initial number of reconstructed rows, lead to similar results.

${ }^{21}$ A. Bartolini, F. Ercolessi, and E. Tosatti, Phys. Rev. Lett. 63, 872 (1989).

${ }^{22}$ Y. Wang, N. S. Hush, and J. R. Reimers, Phys. Rev. B 75, 233416 (2007).

${ }^{23}$ N. Takeuchi, C. T. Chan, and K. M. Ho, Phys. Rev. B 43, 13899 (1991).

${ }^{24}$ Z. Crljen, P. Lazic, D. Sokcevic, and R. Brako, Phys. Rev. B 68, 195411 (2003).

${ }^{25}$ L. Guan, L. Xu, G. Jianxin, J. Litao, Z. Qingxun, and L. Baoting, Solid State Commun. 149, 1561 (2009).

${ }^{26}$ R. Ferrando, G. Barcaro, and A. Fortunelli, Phys. Rev. Lett. 102, 216102 (2009).

${ }^{27}$ S. Olivier, R. Conte, and A. Fortunelli, Phys. Rev. B 77, 054104 (2008).

${ }^{28}$ P. Pyykkö, Angew. Chem., Int. Ed. 43, 4412 (2004).

${ }^{29}$ R. Gaspari et al. (unpublished).

${ }^{30}$ M.-T. Nguyen, C. A. Pignedoli, M. Treier, R. Fasel, and D. Passerone, Phys. Chem. Chem. Phys. 12, 992 (2010).

${ }^{31}$ S. K. M. Henze, O. Bauer, T. L. Lee, M. Sokolowski, and F. S. Tautz, Surf. Sci. 601, 1566 (2007).

${ }^{32}$ S. Grimme, J. Comput. Chem. 27, 1787 (2006). 